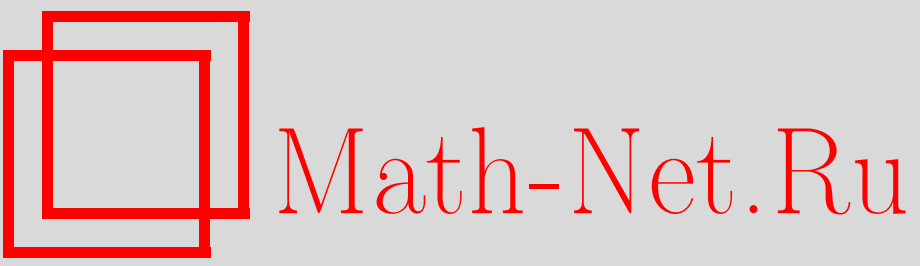

В. С. Минеев, Физика самосопряженных расширений: одномерная задача рассеяния для кулоновского потенциала, ТМФ, 2004, том 140, номер 2, 310-328

DOI: https://doi.org/10.4213/tmf92

Использование Общероссийского математического портала Math-Net.Ru подразумевает, что вы прочитали и согласны с пользовательским соглашением

http://www . mathnet.ru/rus/agreement

Параметры загрузки:

IP : 54.84 .234 .179

26 апреля 2023 г., 10:26:51 
ТЕОРЕТИЧЕСКАЯ

И МАТЕМАТИЧЕСКАЯ

ФИЗИКА

Том 140, № 2

август, 2004

(C) 2004 г.

В.С. Минеев*

\title{
ФИЗИКА САМОСОПРЯЖЕННЫХ РАСШИРЕНИЙ: ОДНОМЕРНАЯ ЗАДАЧА РАССЕЯНИЯ ДЛЯ КУЛОНОВСКОГО ПОТЕНЦИАЛА
}

\begin{abstract}
В работе рассматривается одномерная одноцентровая задача рассеяния на всей прямой с исходным потенциалом $\alpha|x|^{-1}$, которая сводится к поиску допустимых самосопряженных расширений. Использование в качестве необходимых условий законов сохранения в точке сингулярности наряду с учетом аналитической структуры фундаментальных решений позволяет получить точные выражения для волновых функций (т.е. для граничных условий), коэффициентов рассеяния и сингулярных поправок к потенциалу, а также соответствующий спектр связанных состояний. При этом оказывается, что точечные $\delta$-образные поправки к потенциалу должны присутствовать в обязательном порядке при любом выборе допустимого самосопряженного расширения, причем вид этих поправок соответствует виду перенормировочных членов, получаемых в квантовой электродинамике. Таким образом, предложенный метод показывает однозначную связь граничных условий, коэффициентов рассеяния и $\delta$-образных добавок к потенциалу и в целом демонстрирует возможности, которые возникают при анализе самосопряженных расширений соответствующего оператора Гамильтона. При этом в части, касающейся теории перенормировок, он может рассматриваться как обобщение метода перенормировок Боголюбова, Парасюка и Хеппа.
\end{abstract}

Ключевые слова: кулоновское взаимодействие, точечное взаимодействие, задача рассеяния, самосопряженные расширения, перенормировки.

Исследование сингулярных взаимодействий в квантовой теории имеет уже достаточно длительную историю. Одной из первых работ, посвяшенных сингулярным потенциалам, является работа [1], а исследование уравнения Шредингера с $\delta$-образными особенностями восходит к работе [2]. В последующие годы это направление исследования породило обширную литературу (см. [3]), причем серьезный математический анализ возникаюших при этом проблем был выполнен сравнительно недавно [4]. Относительно недавно было проведено, в частности, и исследование трехмерного уравнения Шредингера, в котором присутствуют одновременно кулоновское и точечное взаимодействия, причем кулоновское взаимодействие рассматривается как возмушение трехмерной одноцентровой задачи [5] (основы подхода, развитого в работах [5], были заложены еще в работе [6]).

* Научно-исследовательский институт ядерной физики Московского государственного университета, Москва, Россия. E-mail: mineev@theory.sinp.msu.ru 
В абсолютно подавляющем большинстве работ (как и в работах [5]) рассматривался именно трехмерньй случай и проводилось парциальное разложение волновых функций (или соответствуюшего пространства $L^{2}\left(\mathbb{R}^{3}\right)$ ) по отношению к угловому моменту. В свою очередь, приведенная радиальная волновая функция однозначно определялась граничным условием $\psi(0)=0$, что соответствует существованию однопараметрического семейства самосопряженных операторов. При этом наиболее подробно изучалась угловая компонента волновой функции.

Следует отметить, что впервые определение гамильтониана как самосопряженного оператора в пространстве $L^{2}\left(\mathbb{R}^{3}\right)$ было дано в работе [7].

Одномерный случай исследован гораздо меньше трехмерного. В одномерном случае, в отличие от двух- и трехмерных, необходимо рассматривать оба линейно независимых решения, что при наличии сингулярности приводит к сушествованию четырехпараметрического семейства самосопряженных расширений в пространстве $L^{2}(\mathbb{R})$. А это, в свою очередь, может приводить к возможности сушествования дополнительных точечных взаимодействий.

В настояшей работе рассматривается одномерная одноцентровая задача рассеяния на всей прямой с исходным потенциалом $\alpha|x|^{-1}$. Общее решение стационарного уравнения Шредингера с кулоновским потенциалом известно, и задача сводится к поиску самосопряженных расширений или, в более узком толковании, к поиску граничных условий в точке сингулярности, удовлетворяющих общим принципам квантовой теории.

Отметим здесь, кстати, что различным аспектам кулоновского взаимодействия посвящено необозримое количество работ. Однако количество работ, посвяшенных математическим проблемам, связанным с наличием неинтегрируемой сингулярности в точке взаимодействия, не слишком велико. В данной статье мы ограничимся обзором только обших работ, относяшихся к рассматриваемым проблемам. Более полный обзор позднейших публикаций, посвященных данной тематике, будет приведен в следуюшей статье. Добавим здесь только, что в подавляюшем большинстве работ, посвяшенных одномерной кулоновской задаче, рассматривались лишш проблемы связанных состояний и их волновых функций. Чуть ли не единственным исключением (причем посвященным несколько другому - кулонообразному - сингулярному взаимодействию) является работа [8], вызвавшая возражения, высказанные в статье [9] (ответ на эти возражения см. в работе [10]).

Для решения указанной выше проблемы выбора граничных условий (т.е. для конкретизации значений параметров, определяющих соответствующее самосопряженное расширение) в качестве необходимых условий в точке сингулярности $x=0$ будем использовать условия сохранения плотности вероятности и потока вероятности. Такой подход в сочетании с использованием метода вариации постоянных, аналитической структуры волновых функций и теории обобшенных функций позволяет полностью решить одномерную задачу рассеяния для кулоновского потенциала. При этом оказывается, что указанные условия в обязательном порядке приводят к необходимости включения в потенциал сингулярных $\delta$-образных добавок.

Следует отметить, что, вообще говоря, допустима возможность существования целого ряда наборов указанных параметров, т.е. существует неоднозначность в выборе допустимых решений и гамильтонианов. Такая неоднозначность на языке теории опера- 
торов соответствует тому, что рассматриваемьй неограниченный симметрический (т.е. эрмитов) оператор не является самосопряженным на всей оси и допускает различные самосопряженные расширения (см. [11], [12]). На факт допустимости нескольких типов решений, вероятно, впервые было обращено внимание в работах [13], которые, в свою очередь, привели к анализу связи топологии и квантовой статистики и соответственно к исследованию зависимости от выбора граничных условий в точке сингулярности описания бозонов, фермионов и парачастиц (см., например, [14]).

Рассмотрим стационарное одномерное уравнение Шредингера

$$
\psi^{\prime \prime}(x)+\left[k^{2}-u(x)\right] \psi(x)=0
$$

с потенциалом

$$
u(x)=\varepsilon(x) \frac{\alpha}{x}, \quad x, \alpha \in \mathbb{R},
$$

где $\varepsilon(x)-$ знаковая функция

$$
\varepsilon(x)=\theta(x)-\theta(-x)
$$

$(\theta(x)$ - характеристическая (ступенчатая) функция Хевисайда). Будем строить обшее решение уравнения (1), используя функции Уиттекера, соответствуюшие задаче рассеяния, т.е. выберем решения в виде фундаментальной пары (см. приложение)

$$
\begin{aligned}
& \psi_{+}^{+, W}(k, x)=e^{\pi \alpha /(4 k)} W_{-\frac{i \alpha}{2 k}, \frac{1}{2}}(-2 i k x) \\
& \psi_{+}^{-, V}(k, x)=-e^{\pi \alpha /(4 k)} V_{-\frac{i \alpha}{2 k}, \frac{1}{2}}(-2 i k x)
\end{aligned}
$$

для положительной полуоси и

$$
\begin{aligned}
& \psi_{-}^{+, W}(k, x)=e^{-\pi \alpha /(4 k)} W_{\frac{i \alpha}{2 k}, \frac{1}{2}}(-2 i k x), \\
& \psi_{-}^{-, V}(k, x)=-e^{-\pi \alpha /(4 k)} V_{\frac{i \alpha}{2 k}, \frac{1}{2}}(-2 i k x)
\end{aligned}
$$

для отрицательной полуоси. Функции (4), (5), как отмечено в приложении, характеризуются асимптотическим поведением:

$$
\begin{aligned}
& \lim _{x \rightarrow+\infty} \psi_{+}^{+, W}(k, x)=e^{i(k x-\alpha \ln (2 k x) /(2 k))}\left[1+O\left(\frac{1}{-2 i k x}\right)\right], \\
& \lim _{x \rightarrow+\infty} \psi_{+}^{-, V}(k, x)=e^{-i(k x-\alpha \ln (2 k x) /(2 k))}\left[1+O\left(\frac{1}{2 i k x}\right)\right], \\
& \lim _{x \rightarrow-\infty} \psi_{-}^{+, W}(k, x)=e^{i(k x+\alpha \ln (2 k x) /(2 k))}\left[1+O\left(\frac{1}{-2 i k x}\right)\right], \\
& \lim _{x \rightarrow-\infty} \psi_{-}^{-, V}(k, x)=e^{-i(k x+\alpha \ln (2 k x) /(2 k))}\left[1+O\left(\frac{1}{2 i k x}\right)\right],
\end{aligned}
$$

а их вронскианы (см. формулу (П.5)) имеют вид

$$
\begin{aligned}
& \mathcal{W}\left\{\psi_{+}^{+, W}(k, x), \psi_{+}^{-, V}(k, x)\right\}=-2 i k \\
& \mathcal{W}\left\{\psi_{-}^{+, W}(k, x), \psi_{-}^{-, V}(k, x)\right\}=-2 i k .
\end{aligned}
$$


Выбор решений (4), (5) с асимптотическим поведением (6) фиксирует поведение решений уравнения (1) на бесконечности. Однако, как было отмечено выше, оператор Шредингера (1) с сингулярным потенциалом (2) (как и оператор импульса) является примером замкнутого симметрического, но не самосопряженного оператора (см. [11], [12]) и определяется четырехпараметрическим семейством самосопряженных расширений (см. [15], а также [4]), которое может быть сведено к трехпараметрическому семейству [16]. Конкретный выбор самосопряженного расширения, как известно [12], определяет соответствующую физику. В свою очередь, с точки зрения физики для определения полного набора состояний необходимо фиксировать поведение волновых функций (и оператора) вблизи сингулярных точек, что и определяет расширение оператора до самосопряженного.

Прежде чем фиксировать поведение решений в нуле, напомним стандартное определение коэффициентов отражения и прохождения. Обычно в простейших случаях, соответствующих "хорошим" (см., например, [17]) потенциалам (т.е. в случаях, когда оператор самосопряжен, или, иначе говоря, его индексы дефекта равны $(0,0)$ (см. [7], а также [12], [4])), в качестве фундаментальной пары берут пару фундаментальных решений (или решений Йоста) $f_{1}(k, x)$ и $f_{1}(-k, x)$, характеризуюшихся асимптотическим поведением

$$
\lim _{x \rightarrow+\infty}\left[e^{-i k x} f_{1}(k, x)\right]=1,
$$

или $f_{2}(k, x)$ и $f_{2}(-k, x)$ с поведением

$$
\lim _{x \rightarrow-\infty}\left[e^{i k x} f_{2}(k, x)\right]=1 .
$$

Так как любое третье решение есть комбинация двух линейно независимых решений, то можно написать, что, например,

$$
\begin{aligned}
& f_{2}(k, x)=c_{11}(k) f_{1}(k, x)+c_{12}(k) f_{1}(-k, x), \\
& f_{1}(k, x)=c_{21}(k) f_{2}(-k, x)+c_{22}(k) f_{2}(k, x) .
\end{aligned}
$$

Рассматривая теперь предельные значения соотношений (10), легко увидеть, что (10а) представляет собой решение уравнения (1), которое при $x \rightarrow-\infty$ стремится к $e^{-i k x}, \mathrm{a}$ при $x \rightarrow+\infty-$ к линейной комбинации $c_{11} e^{i k x}+c_{12} e^{-i k x}$. Таким образом, решение (10a) соответствует задаче рассеяния, в которой из $x=+\infty$ (R-случай) на рассеиваюший потенциал падает волна с амплитудой $c_{12}$, частично отражаюшаяся с амплитудой $c_{11}, \mathrm{a}$ частично проходящая на $-\infty$ с амплитудой, равной 1. Это означает, что коэффициенты отражения и прохождения имеют вид

$$
\begin{aligned}
& R_{\mathrm{R}}(k)=\frac{c_{11}(k)}{c_{12}(k)} \\
& T_{\mathrm{R}}(k)=\frac{1}{c_{12}(k)} .
\end{aligned}
$$

Аналогично решение (10б) описывает задачу рассеяния с волной, падающей слева (L-случай), и коэффициентами

$$
\begin{aligned}
& R_{\mathrm{L}}(k)=\frac{c_{22}(k)}{c_{21}(k)} \\
& T_{\mathrm{L}}(k)=\frac{1}{c_{21}(k)}
\end{aligned}
$$


В рассматриваемом в настоящей работе случае осуществление такой программы имеет ту трудность, что функции Уиттекера (П.6) и (П.7) (см. приложение) имеют при $z=0$ регулярную особую точку, являюшуюся логарифмической точкой ветвления, т.е. связь функций $f_{1}(k, x)$ и $f_{1}(-k, x)$ (и соответственно $f_{2}(k, x)$ и $f_{2}(-k, x)$ ) неоднозначна, причем мы заранее не знаем, на каком листе окажутся отраженная и проходящая волны. Однако тот факт, что сушествует аналитическое продолжение для произвольного решения уравнения (1) (см. приложение), причем обе функции $W_{p, m}\left(z e^{2 i \pi s}\right)$ и $V_{p, m}\left(z e^{2 i \pi s}\right)$ являются решениями уравнения (1), позволяет произвольное решение уравнения Шредингера (1) представить в виде их линейной комбинации. В нашем случае это означает, что при $x>0$ справедливо выражение

$$
\psi_{+}(k, x)=\alpha_{2}^{+}(k) \psi_{+}^{-, V}(k, x)+\beta_{2}^{+}(k) \psi_{+}^{-, V}\left(e^{2 i \pi s} k, x\right) .
$$

Соответственно на отрицательной полуоси

$$
\psi_{-}(k, x)=\alpha_{2}^{-}(k) \psi_{-}^{-, V}\left(k, e^{2 i \pi r} x\right)+\beta_{2}^{-}(k) \psi_{-}^{-, V}\left(e^{2 i \pi s} k, e^{2 i \pi r} x\right) .
$$

При этом в выражениях (13), (14) необходимо учитывать, что при прохождении переменной $x$ через точку сингулярности $x=0$ могут, вообще говоря, изменяться коэффициенты при соответствуюших волновых функциях (это явление связано с показателями регулярной особой точки; см. приложение, а также монографию [18]). Вводя обозначения

$$
\begin{aligned}
& \alpha_{2}^{-}(k)=\alpha_{2}^{+}(k) Q_{1}=\alpha_{2}(k) Q_{1}, \\
& \beta_{2}^{-}(k)=\beta_{2}^{+}(k) Q_{2}=\beta_{2}(k) Q_{2},
\end{aligned}
$$

можно записать решение уравнения (1) на всей прямой в виде

$$
\begin{aligned}
\psi(x)= & \alpha_{2}(k)\left[\psi_{+}^{-, V}(k, x) \theta(x)+Q_{1} \psi_{-}^{-, V}\left(k, e^{2 i \pi r} x\right) \theta(-x)\right]+ \\
& +\beta_{2}(k)\left[\psi_{+}^{-, V}\left(e^{2 i \pi s} k, x\right) \theta(x)+Q_{2} \psi_{-}^{-, V}\left(e^{2 i \pi s} k, e^{2 i \pi r} x\right) \theta(-x)\right] .
\end{aligned}
$$

Формулы аналитического продолжения функций Уиттекера (П.30) могут быть представлены в виде

$$
V_{p, \frac{1}{2}}\left(e^{2 i \pi s} z\right)=b_{s}^{V}(p) V_{p, \frac{1}{2}}(z)+b_{s}^{W}(p) W_{p, \frac{1}{2}}(z)
$$

где

$$
\begin{aligned}
& b_{s}^{V}(p)=-s e^{2 i \pi p}+(s+1) \\
& b_{s}^{W}(p)=-s \frac{2 i \pi e^{i \pi p}}{\Gamma(p) \Gamma(1+p)}
\end{aligned}
$$

Это позволяет переписать выражение (17) в форме

$$
\begin{aligned}
\psi(x)= & {\left[\alpha_{+}^{-}(k) \psi_{+}^{-, V}(k, x)+\alpha_{+}^{+}(k) \psi_{+}^{+, W}(k, x)\right] \theta(x)+} \\
& +\left[\alpha_{-}^{-}(k) \psi_{-}^{-, V}(k, x)+\alpha_{-}^{+}(k) \psi_{-}^{+, W}(k, x)\right] \theta(-x),
\end{aligned}
$$


где

$$
\begin{aligned}
& \alpha_{+}^{-}(k)=\alpha_{2}(k)+\beta_{2}(k) b_{s}^{V}\left(-\frac{i \alpha}{2 k}\right), \\
& \alpha_{+}^{+}(k)=-\beta_{2}(k) b_{s}^{W}\left(-\frac{i \alpha}{2 k}\right), \\
& \alpha_{-}^{-}(k)=Q_{1} \alpha_{2}(k) b_{r}^{V}\left(\frac{i \alpha}{2 k}\right)+Q_{2} \beta_{2}(k) b_{r+s}^{V}\left(\frac{i \alpha}{2 k}\right), \\
& \alpha_{-}^{+}(k)=-\left[Q_{1} \alpha_{2}(k) b_{r}^{W}\left(\frac{i \alpha}{2 k}\right)+Q_{2} \beta_{2}(k) b_{r+s}^{W}\left(\frac{i \alpha}{2 k}\right)\right] .
\end{aligned}
$$

Отметим, что хотя выражение (17) соответствует формуле (10б), оно носит совершенно обший характер, волновая функция $\psi(x)(21)$ представляет собой произвольное решение уравнения (1) с потенциалом (2). Соответствуюшее представление может быть получено при построении аналога формулы (10a).

Вернемся теперь к задаче рассеяния, кратко описанной соотношениями (9)-(12). Рассмотрим, например, случай, когда решение $\psi(x)(21)$ является фундаментальным решением с асимптотическим поведением, аналогичным поведению (9б), т.е. положим $\psi(x)=$ $f_{2}(k, x)$. Сравнение выражений $(21)$ и (10а) показывает, что решение, описывающее рассеяние частицы, падающей справа (R-случай), должно иметь вид

$$
\psi(x)=f_{2}(k, x)=\mathcal{A}_{\mathrm{R}}(k) \psi_{+}^{-, V}(k, x) \theta(x)+\mathcal{B}_{\mathrm{R}}(k) \psi_{+}^{+, W}(k, x) \theta(x)+\psi_{-}^{-, V}(k, x) \theta(-x),
$$

а выражения для коэффициентов рассеяния (11) в данном случае приобретают форму

$$
\begin{aligned}
& R_{\mathrm{R}}(k)=\frac{\mathcal{B}_{\mathrm{R}}(k)}{\mathcal{A}_{\mathrm{R}}(k)}, \\
& T_{\mathrm{R}}(k)=\frac{1}{\mathcal{A}_{\mathrm{R}}(k)} .
\end{aligned}
$$

Воспользуемся теперь несколько модифицированным методом вариации постоянных, т.е. будем рассматривать коэффициенты $\alpha_{2}(k)$ и $\beta_{2}(k)$ в выражении (17) (и соответственно $\alpha_{+}^{-}(k), \alpha_{+}^{+}(k), \alpha_{-}^{-}(k), \alpha_{-}^{+}(k)$ в формулах $\left.(22)-(25)\right)$ как величины, зависящие от $x$. Если представить выражения для $\alpha_{2}(k, x)$ и $\beta_{2}(k, x)$ в виде

$$
\begin{aligned}
\alpha_{2}(k, x) & =A_{1}(k) \theta(x)+A_{2}(k) \theta(-x), \\
\beta_{2}(k, x) & =B_{1}(k) \theta(x)+B_{2}(k) \theta(-x),
\end{aligned}
$$

то из сравнения выражений (17)-(25) и (26) следует, что в рассматриваемом случае рассеяния частиц, падающих справа,

$$
\begin{aligned}
& A_{2}(k)=\frac{1}{Q_{1}} \frac{r+s}{s}, \\
& B_{2}(k)=-\frac{1}{Q_{2}} \frac{r}{s},
\end{aligned}
$$


т.e.

$$
\begin{aligned}
& \mathcal{A}_{\mathrm{R}}(k)=A_{1}(k)+B_{1}(k) b_{s}^{V}\left(-\frac{i \alpha}{2 k}\right), \\
& \mathcal{B}_{\mathrm{R}}(k)=-B_{1}(k) b_{s}^{W}\left(-\frac{i \alpha}{2 k}\right)
\end{aligned}
$$

и

$$
\begin{aligned}
\alpha_{2}(k, x) & =\left[\mathcal{A}_{\mathrm{R}}(k)+\mathcal{B}_{\mathrm{R}}(k) \frac{b_{s}^{V}(-i \alpha /(2 k))}{b_{s}^{W}(-i \alpha /(2 k))}\right] \theta(x)+\frac{1}{Q_{1}} \frac{r+s}{s} \theta(-x), \\
\beta_{2}(k, x) & =-\mathcal{B}_{\mathrm{R}}(k) \frac{1}{b_{s}^{W}(-i \alpha /(2 k))} \theta(x)-\frac{1}{Q_{2}} \frac{r}{s} \theta(-x) .
\end{aligned}
$$

Выражения (35), (36) явным образом демонстрируют отличие в определении коэффициентов рассеяния в рассматриваемом здесь сингулярном и простейшем стандартном случаях (ср. формулы (10)-(12) и (17), (35), (36), (27), (28)).

Обычное в методе вариашии постоянных условие отсутствия членов, связанных с дифференцированием коэффициентов при волновых функциях, приводит в нашем случае к требованию отсутствия в производной $f_{2}^{\prime}(k, x)$ члена вида

$$
\left[\mathcal{A}_{\mathrm{R}}(k) \psi_{+}^{-, V}(k, x)+\mathcal{B}_{\mathrm{R}}(k) \psi_{+}^{+, W}(k, x)-\psi_{-}^{-, V}(k, x)\right] \delta(x) .
$$

Разложение в ряд функций $\psi_{+}^{-, V}(k, x), \psi_{+}^{+, W}(k, x)\left(\right.$ и $\left.\psi_{-}^{-, V}(k, x)\right)$ (см. (П.22) и (П.24)) с учетом операторного соотношения $x \delta(x)=0$ и выражений (33), (34) приводит к условию

$$
\begin{aligned}
& e^{\pi \alpha /(4 k)}\left[\left(A_{1}(k)+B_{1}(k) b_{s}^{V}\left(-\frac{i \alpha}{2 k}\right)\right) V_{-\frac{i \alpha}{2 k}, \frac{1}{2}}(0)+B_{1}(k) b_{s}^{W}\left(-\frac{i \alpha}{2 k}\right) W_{-\frac{i \alpha}{2 k}, \frac{1}{2}}(0)\right]- \\
& -e^{-\pi \alpha /(4 k)} V_{\frac{i \alpha}{2 k}, \frac{1}{2}}(0)=0
\end{aligned}
$$

При этом

$$
\begin{aligned}
f_{2}^{\prime}(k, x)= & \alpha_{2}(k, x)\left[\psi_{+}^{-, V^{\prime}}(k, x) \theta(x)+Q_{1} \psi_{-}^{-, V^{\prime}}\left(k, e^{2 i \pi r} x\right) \theta(-x)\right]+ \\
& +\beta_{2}(k, x)\left[\psi_{+}^{-,}{ }^{\prime}\left(e^{2 i \pi s} k, x\right) \theta(x)+Q_{2} \psi_{-}^{-, V^{\prime}}\left(e^{2 i \pi s} k, e^{2 i \pi r} x\right) \theta(-x)\right] .
\end{aligned}
$$

Выражение для плотности тока, определенное стандартным образом,

$$
j(k, x)=\frac{1}{2 i}\left[\psi^{*}(k, x) \psi^{\prime}(k, x)-\psi(k, x) \psi^{* \prime}(k, x)\right],
$$

с учетом вронскианов (7), (8) и (П.31)-(П.33) приобретает вид

$$
j(k)=k\left(-\left|\mathcal{A}_{\mathrm{R}}(k)\right|^{2}+\left|\mathcal{B}_{\mathrm{R}}(k)\right|^{2}\right),
$$

а закон сохранения тока (т.е. условие унитарности) -

$$
\left|\mathcal{A}_{\mathrm{R}}(k)\right|^{2}-\left|\mathcal{B}_{\mathrm{R}}(k)\right|^{2}=1
$$


или на языке коэффициентов рассеяния $(27),(28)$

$$
\left|R_{\mathrm{R}}(k)\right|^{2}+\left|T_{\mathrm{R}}(k)\right|^{2}=1 \text {. }
$$

Отметим, что условие (38) совпадает с условием четного продолжения функции $f_{2}(k, x)$ при прохождении через точку $x=0$, т.е. обеспечивает сохранение вероятности в нуле.

Вычисляя вторую производную функции $\psi(k, x)=f_{2}(k, x)$ и подставляя получившееся выражение в уравнение (1) с потенциалом (2), с учетом выражений $(17),(21),(26)$ получаем

$$
\begin{aligned}
& {\left[\mathcal{A}_{\mathrm{R}}(k) \psi_{+}^{-, V^{\prime}}(k, x)+\mathcal{B}_{\mathrm{R}}(k) \psi_{+}^{+, W^{\prime}}(k, x)-\psi_{-}^{-, V^{\prime}}(k, x)\right] \delta(x)+} \\
& \quad+\mathcal{A}_{\mathrm{R}}(k)\left\{\psi_{+}^{-, V^{\prime \prime}}(k, x)+\left[k^{2}-\varepsilon(x) \frac{\alpha}{x}\right] \psi_{+}^{-, V}(k, x)\right\} \theta(x)+ \\
& \quad+\mathcal{B}_{\mathrm{R}}(k)\left\{\psi_{+}^{+, W^{\prime \prime}}(k, x)+\left[k^{2}-\varepsilon(x) \frac{\alpha}{x}\right] \psi_{+}^{+, W}(k, x)\right\} \theta(x)+ \\
& \quad+\left\{\psi_{-}^{-, V \prime \prime}(k, x)+\left[k^{2}-\varepsilon(x) \frac{\alpha}{x}\right] \psi_{-}^{-, V}(k, x)\right\} \theta(-x)=0 .
\end{aligned}
$$

Из этого выражения видно, что построение решения в виде (17), т.е. согласование решений $(13)$ на $\mathbb{R}_{+}$и $(14)$ на $\mathbb{R}_{-}$в нуле, приводит к появлению дополнительного первого члена в (44). Казалось бы, этот член можно рассматривать как сингулярную добавку к потенциалу, являющуюся проявлением граничных условий в нуле. Однако (и последуюшие расчеты это подтверждают) при этом нельзя быть уверенным в сохранении тока и выполнении условия (38). Поэтому введем в (44) взаимно компенсируюшие друг друга члены вида $\alpha_{2}(k, x) \psi_{i}(k, x) \delta V_{i}(k, x)$ и $\beta_{2}(k, x) \psi_{i}(k, x) \delta V_{i}(k, x)$ (где $\left.\psi_{i}(k, x)=\psi_{+}^{-, V}(k, x), \psi_{+}^{+, W}(k, x), \psi_{-}^{-, V}(k, x), \psi_{-}^{+, W}(k, x)\right)$. Появление этих членов означает, что функции $\psi_{i}(k, x)$ должны удовлетворять уравнению Шредингера с учетом граничных условий, т.е. уравнения для них приобретают вид

$$
\psi_{i}^{\prime \prime}(k, x)+\left[k^{2}-\varepsilon(x) \frac{\alpha}{x}-\delta V_{i}(k, x)\right] \psi_{i}(k, x)=0 .
$$

Некоторые математические детали возможности сосуществования кулоновского и точечного потенщиалов (в частности, в смысле КЛМН-теоремы (теоремы Като-ЛайонсаМильграма-Нельсона)) см. в книгах [4], а также [12].

Будем искать сингулярные добавки $\delta V_{i}(k, x)$ в виде

$$
\delta V_{i}(k, x)=v_{i} \frac{\psi_{i}^{\prime}(k, x)}{\psi_{i}(k, x)} \delta(x),
$$

где $v_{i}=v_{+}^{-, V}, v_{-}^{-, V}, v_{-}^{+, W}, v_{+}^{+, W}$ - параметры для соответствующих $\psi_{i}(k, x)$. Форма (46) для $\delta V_{i}(k, x)$ делает их, с одной стороны, аналогичными первому члену формулы (44), а с другой - представляет сингулярные добавки к потенциалу в виде логарифмической производной (см., например, [19]).

Объединяя соотношения (45) и учитывая выражения (26), (33)-(36), получаем, что в случае рассеяния частиц, падаюших справа, наложение граничных условий при $x=0$ 
приводит к тому, что соответствуюшая волновая функция $f_{2}(k, x)$ должна удовлетворять уравнению

$$
f_{2}^{\prime \prime}(k, x)+\left[k^{2}-\varepsilon(x) \frac{\alpha}{x}-\delta V_{f_{2}}(k, x)\right] f_{2}(k, x)=0,
$$

где

$$
\begin{aligned}
\delta V_{f_{2}}(k, x)= & {\left[\frac{\mathcal{A}_{\mathrm{R}}(k) v_{+}^{-, V} \psi_{+}^{-, V^{\prime}}(k, x)+\mathcal{B}_{\mathrm{R}}(k) v_{+}^{+, W} \psi_{+}^{+, W '}(k, x)}{\mathcal{A}_{\mathrm{R}}(k) \psi_{+}^{-, V}(k, x)+\mathcal{B}_{\mathrm{R}}(k) \psi_{+}^{+, W}(k, x)} \theta(x)+\right.} \\
& \left.+v_{-}^{-, V} \frac{\psi_{-}^{-, V^{\prime}}(k, x)}{\psi_{-}^{-, V}(k, x)} \theta(-x)\right] \delta(x)
\end{aligned}
$$

при условии отсутствия первого члена в соотношении (44). В отличие от случая условия (38), структура разложения функций $\psi_{i}^{\prime}(k, x)(\Pi .23)$ и (П.25) с учетом операторного соотношения $x \delta(x)=0$ приводит к необходимости выполнения двух условий:

$$
\begin{aligned}
e^{\pi \alpha /(4 k)} & {\left[\left(A_{1}(k)+B_{1}(k) b_{s}^{V}\left(-\frac{i \alpha}{2 k}\right)\right)\left(1+v_{+}^{-, V}\right) V_{-\frac{i \alpha}{2 k}, \frac{1}{2}}^{\prime}(0)+\right.} \\
+ & \left.B_{1}(k) b_{s}^{W}\left(-\frac{i \alpha}{2 k}\right)\left(1+v_{+}^{+, W}\right) W_{-\frac{i \alpha}{2 k}, \frac{1}{2}}^{\prime}(0)\right]- \\
- & e^{-\pi \alpha /(4 k)}\left(1-v_{-}^{-, V}\right) V_{\frac{i \alpha}{2 k}, \frac{1}{2}}^{\prime}(0)=0 \\
e^{\pi \alpha /(4 k)} & {\left[\left(A_{1}(k)+B_{1}(k) b_{s}^{V}\left(-\frac{i \alpha}{2 k}\right)\right)\left(1+v_{+}^{-, V}\right) \frac{1}{\Gamma(-i \alpha /(2 k))}+\right.} \\
+ & \left.B_{1}(k) b_{s}^{W}\left(-\frac{i \alpha}{2 k}\right)\left(1+v_{+}^{+, W}\right) \frac{1}{\Gamma(i \alpha /(2 k))}\right]- \\
- & e^{-\pi \alpha /(4 k)}\left(1-v_{-}^{-, V}\right) \frac{1}{\Gamma(i \alpha /(2 k))}=0 .
\end{aligned}
$$

Условие (50) обеспечивает отсутствие в разложении членов вида $\delta(x) \ln x$.

Проведенное построение нуждается в некоторых комментариях.

Прежде всего следует отметить, что из выражения (44) и рассуждений, приводяших к виду (48) сингулярной добавки к потенциалу, видно, что условие (38) (помимо того, что оно, как указывалось выше, обеспечивает непрерывность продолжения функции $f_{2}(k, x)$ при прохождении через нуль) одновременно является условием именно $\delta$-образного вида сингулярной добавки (48). Невыполнение условия (38), в частности, может приводить к появлению в уравнении (1) членов, пропорциональных $\delta^{\prime}(x)$.

В целом сам факт присутствия в уравнении (47) сингулярной добавки (48) требует пояснения. Суть дела состоит в том, что с точки зрения теории обобщенных функций появление такого члена совершенно естественно. И именно продолжение непрерывного линейного функционала (в нашем случае - гамильтониана), заданного на $\mathcal{J}(\mathbb{R} \backslash 0)=$ $\{f \in \mathcal{J} \mid \operatorname{supp} f \subset \mathbb{R} \backslash 0\}$, на все пространство $\mathcal{J}(\mathbb{R})$ называется "перенормировкой” данного функционала [11]. При этом перенормировка функционала имеет носитель, равньй $\{0\}$, т.е. представляет собой, вообще говоря, бесконечный ряд вида $\sum_{\alpha} c_{\alpha} D^{\alpha} \delta(x)$, где $c_{\alpha}$ - некоторые свободные константы, а $D^{\alpha}$-производная в смысле обобшенных функций 
(или слабая производная) (см. [11]). Как раз таким образом интерпретировались перенормировки в теории $R$-операции Боголюбова, Парасюка и Хеппа [20] (см. также [21]). Однако в теории Боголюбова, Парасюка и Хеппа рассматривались только перенормировки членов ряда фейнмановского разложения, причем для вычисления констант $c_{\alpha}$ (которые выступали в роли, например, перенормировок массы и заряда) использовалась регуляризация Паули-Вилларса (см., например, [21]). В нашем случае, как указывалось вьше, в качестве соответствующего функционала рассматривается весь гамильтониан, а перенормировочные константы $c_{\alpha}$ определяются совершенно иным способом. Как видно из выражения (48), единственный присутствуюший в рассматриваемом случае коэффициент $c_{0}$ является функцией параметров $A_{i}, B_{i}$ и $v_{i}$. В свою очередь, эти параметры определяются уравнениями (38), (49) и (50), полученными в результате интегрирования выражений, в которые входят произведения обобщенных функций $\theta( \pm x) \delta(x)$. Вообще говоря, произведение нескольких обобшенных функций не является хорошо определенной интегрируемой функцией. Тем не менее для основных функций, непрерывных в точке сингулярности, произведение $\theta( \pm x) \delta(x)$ определено (например, в смысле несобственного предельного перехода, см. [21]); обычно принято полагать для таких основных функций $\theta( \pm x) \delta(x)=(1 / 2) \delta(x)$. В нашем случае основная функция не определена в точке сингулярности, и произведение обобшенных функций $\theta( \pm x)$ и $\delta(x)$ требует доопределения. Иначе говоря, возникает проблема определения, вообще говоря, произвольных коэффициентов $c_{ \pm}$в соотношениях $\int f(x) \theta( \pm x) \delta(x) d x=c_{ \pm} f\left(0^{ \pm}\right)$. Но уравнения (38), (49) и (50) как раз и являются уравнениями, определяющими коэффициенты $c_{ \pm}$, в роли которых выступают комбинации параметров $A_{i}, B_{i}$ и $v_{i}$. Таким образом, решив систему этих уравнений, мы и определим произведения $\theta( \pm x) \delta(x)$, что, в свою очередь, определит перенормировочные $\delta$-образные добавки в выражении (48).

Решая систему уравнений (38), (49), (50) относительно, например, $A_{1}(k), B_{1}(k)$ и $v_{-}^{-, V}$ и используя выражения $(33),(34)$, получаем обшее решение в виде

$$
\begin{aligned}
\mathcal{A}_{\mathrm{R}}(k) & =\frac{4 \pi k\left(1+v_{+}^{+, W}\right)}{\alpha\left(2+v_{+}^{-, V}+e^{\pi \alpha / k} v_{+}^{-, V}+v_{+}^{+, W}-e^{\pi \alpha / k} v_{+}^{+, W}\right) \Gamma^{2}(i \alpha /(2 k))}, \\
\mathcal{B}_{\mathrm{R}}(k) & =\frac{\left(1+e^{\pi \alpha / k}\right)\left(1+v_{+}^{-, V}\right)}{e^{\pi \alpha /(2 k)}\left(2+v_{+}^{-, V}+e^{\pi \alpha / k} v_{+}^{-, V}+v_{+}^{+, W}-e^{\pi \alpha / k} v_{+}^{+, W}\right)}, \\
v_{-}^{-, V} & =\frac{-4-3 v_{+}^{-, V}-e^{\pi \alpha / k} v_{+}^{-, V}-3 v_{+}^{+, W}+e^{\pi \alpha / k} v_{+}^{+, W}-2 v_{+}^{-, V} v_{+}^{+, W}}{-2-v_{+}^{-, V}-e^{\pi \alpha / k} v_{+}^{-, V}-v_{+}^{+, W}+e^{\pi \alpha / k} v_{+}^{+, W}} .
\end{aligned}
$$

Сразу обрашает на себя внимание отсутствие в формулах (51)-(53) коэффициентов аналитического продолжения $b_{s}^{V}(-i \alpha /(2 k))$ и $b_{s}^{W}(-i \alpha /(2 k))$ и, что наиболее существенно, из формулы (53) видно, что ни при каких значениях импульса $k$ все три коэффициента $v_{i}$ не могут одновременно обращаться в нуль. Это означает, что $\delta$-образные добавки (т.е. перенормировочные контрчлены) обязательно должны присутствовать в уравнениях (45), (47).

Подстановка выражений $(51),(52)$ в формулу (26) дает при $x \rightarrow 0^{+}$предельное значение

$$
f_{2}\left(k, 0^{+}\right)=-\frac{2 i k}{\alpha \Gamma(i \alpha /(2 k))} e^{-\pi \alpha /(4 k)},
$$


совпадаюшее (что соответствует условию $(38)$ ) с пределом $f_{2}\left(k, 0^{-}\right)$. Таким образом, имеем

$$
\left|f_{2}(k, 0)\right|^{2}=\frac{2 k}{\pi \alpha} e^{-\pi \alpha /(2 k)} \operatorname{sh}\left(\frac{\pi \alpha}{2 k}\right)
$$

при любом наборе допустимых значений $v_{i}$, т.е. функции $f_{2}(k, x)$, удовлетворяюшие системе уравнений (38), (49), (50), действительно (как отмечалось выше) хорошо определены и непрерывны в точке сингулярности.

Решения (51)-(53) при наложении ряда дополнительных условий описывают выбор допустимых самосопряженных расширений. Рассмотрим некоторые из них.

1. Будем искать значения $v_{i}$, при которых оператор Шредингера действителен. Будем считать при этом, что параметры $v_{i}$ тоже действительны.

Действительность сингулярной добавки $\delta V_{i}(k, x)(46)$, определенной на отрицательной полуоси, приводит к условию

$$
v_{-}^{-, V}=0
$$

Используя связь между $v_{+}^{+, W}$ и $v_{+}^{-, V}$, получаюшуюся из формулы (53), и накладывая условие действительности сингулярной добавки (48) на положительной полуоси, получаем, что при значениях параметров (56) и

$$
\begin{aligned}
& v_{+}^{-, V}=\frac{-\pi+2 \arg (2 k)+\pi \operatorname{cth}(\pi \alpha /(2 k))}{-2 \arg (2 k)-\pi \operatorname{cth}(\pi \alpha /(2 k))}, \\
& v_{+}^{+, W}=\frac{2\left(\pi+\arg (2 k)-e^{\pi \alpha / k} \arg (2 k)\right)}{\left(-1+e^{\pi \alpha / k}\right)(\pi+2 \arg (2 k))}
\end{aligned}
$$

сингулярная добавка (48) действительна и имеет вид

$$
\delta V_{f_{2}}(k, x)=-2 \alpha\left[2 \gamma_{\mathrm{E}}+\ln \left(2 \sqrt{k^{2}} x\right)+\operatorname{Re} \psi\left(\frac{i \alpha}{2 k}\right)\right] \theta(x) \delta(x)
$$

(здесь $\gamma_{\mathrm{E}}$ - постоянная Эйлера, $\psi(z)=\Gamma^{\prime}(z) / \Gamma(z)$ - дигамма-функция; при вычислении формулы $(59)$, как и ранее, учтено равенство $x \delta(x)=0)$.

Имеет смысл обратить внимание на некоторые свойства добавки (59). Сразу видно, что при выключении взаимодействия $(\alpha=0)$ добавка (59), естественно, также обрашается в нуль. Кроме того, общий знак "минус" в выражении (59) показывает, что поправка к кулоновскому потенциалу $\delta V_{f_{2}}(k, x)$ ослабляет, "компенсирует" исходное взаимодействие $\alpha|x|^{-1}$. Используя представление для $\operatorname{Re} \psi(i \alpha /(2 k))$ в виде ряда (П.35), можно переписать выражение (59) в форме

$$
\begin{gathered}
\delta V_{f_{2}}(k, x)=-2 \alpha\left[\gamma_{\mathrm{E}}+\ln \left(2 \sqrt{k^{2}} x\right)+\frac{\alpha^{2}}{4 k^{2}} \sum_{n=1}^{\infty} \frac{1}{n} \frac{1}{n^{2}+\alpha^{2} /\left(4 k^{2}\right)}\right] \theta(x) \delta(x), \\
-\infty<\frac{\alpha}{2 k}<\infty
\end{gathered}
$$

аналогичной виду фейнмановского разложения. 
Представляет интерес также асимптотическое разложение $\operatorname{Re} \psi(i \alpha /(2 k))$, соответствуюшее низкоэнергетическому случаю. Использование разложения (П.36) дает для $\delta V_{f_{2}}(k, x)$ выражение

$$
\delta V_{f_{2}}(k, x) \sim-2 \alpha\left[2 \gamma_{\mathrm{E}}+\ln (\alpha x)+\sum_{n=1}^{\infty} \frac{(-1)^{n-1} B_{2 n}}{2 n(\alpha /(2 k))^{2 n}}\right] \theta(x) \delta(x), \quad \alpha \gg 2 k
$$

(здесь $B_{2 n}$ - числа Бернулли).

Для случая рассеяния $\arg (2 k)=0$, подставляя выражения (56)-(58) в (51) и (52), имеем

$$
\begin{aligned}
\mathcal{A}_{\mathrm{R}}^{(s)}(k) & =-\frac{2 \pi k}{\alpha} \frac{1}{\Gamma^{2}(i \alpha /(2 k))} \operatorname{cth}\left(\frac{\pi \alpha}{2 k}\right), \\
\mathcal{B}_{\mathrm{R}}^{(s)}(k) & =-\operatorname{sh}\left(\frac{\pi \alpha}{2 k}\right),
\end{aligned}
$$

откуда

$$
\begin{aligned}
& T_{\mathrm{R}}(k)=-\frac{\alpha}{2 \pi k} \Gamma^{2}\left(\frac{i \alpha}{2 k}\right) \operatorname{th}\left(\frac{\pi \alpha}{2 k}\right), \\
& R_{\mathrm{R}}(k)=\frac{\alpha}{2 \pi k} \Gamma^{2}\left(\frac{i \alpha}{2 k}\right) \operatorname{th}\left(\frac{\pi \alpha}{2 k}\right) \operatorname{sh}\left(\frac{\pi \alpha}{2 k}\right),
\end{aligned}
$$

т.е.

$$
\begin{aligned}
\left|T_{\mathrm{R}}(k)\right|^{2} & =\operatorname{sech}^{2}\left(\frac{\pi \alpha}{2 k}\right), \\
\left|R_{\mathrm{R}}(k)\right|^{2} & =\operatorname{th}^{2}\left(\frac{\pi \alpha}{2 k}\right),
\end{aligned}
$$

что обеспечивает вьполнение условия унитарности (43).

Связанным состояниям соответствуют значения импульса, расположенные на положительной мнимой оси, т.е. следует положить $\arg (2 k)=\pi / 2$. В этом случае

$$
\mathcal{A}_{\mathrm{R}}^{(b)}(k)=\frac{2 \pi k}{\alpha} \frac{1}{\Gamma^{2}(i \alpha /(2 k))} e^{\pi \alpha /(2 k)} \operatorname{cosech}\left(\frac{\pi \alpha}{2 k}\right) .
$$

Положение связанных состояний определяется простыми полюсами коэффициента $T_{\mathrm{R}}(k)$, т.е. простыми нулями величины $(68)$. Так как $(\Gamma(z))^{-1}-$ целая функция, обладаюшая простыми нулями при $z=-n$, то легко видеть, что выражение (68) имеет простые нули при

$$
\frac{i \alpha}{2 k}=-n,
$$

т.е. связанные состояния существуют при $\alpha<0$ и имеют отрицательную энергию

$$
E=-\frac{\alpha^{2}}{4 n^{2}} .
$$

Таким образом, рассматриваемый случай приводит к старому известному боровскому выражению для кулоновских уровней энергии. Но при этом потенциал не является 
точно кулоновским - уровни энергии (70) имеют место при наличии дополнительного точечного взаимодействия (59). Более того, поправки (59), записанные в форме (60), (61), чрезвычайно похожи на поправки, возникаюшие в квантовой электродинамике (КЭД) при учете таких явлений, как перенормировки, поляризация вакуума, вакуумные флуктуации и т.д. (причем указанные поправки КЭД обычно принято интерпретировать как релятивистские (см., например, [22])). Сушественно, что полученные выражения (и, в частности, формула (59)) являются точным результатом, полученным вне рамок каких-либо приближений.

2. Случай полной прозрачности. Условие (38) (являюшееся, как отмечалось вьше, условием четного продолжения волновой функции при прохождении через точку сингулярности) можно переписать в виде

$$
R_{\mathrm{R}}(k)-T_{\mathrm{R}}(k) e^{-\pi \alpha /(2 k)}=\frac{\Gamma(i \alpha /(2 k))}{\Gamma(-i \alpha /(2 k))} .
$$

Если положить

$$
R_{\mathrm{R}}(k)=0
$$

TO

$$
T_{\mathrm{R}}(k)=e^{\pi \alpha /(2 k)} \frac{\Gamma(i \alpha /(2 k))}{\Gamma(-i \alpha /(2 k))},
$$

т.e.

$$
\left|T_{\mathrm{R}}(k)\right|^{2}=e^{\pi \alpha / k},
$$

и условие унитарности (43) при действительных значениях импульса $k$ и $\alpha \neq 0$ не выполняется. Таким образом, ни при каких значениях параметров $v_{i}$ одномерньй кулоновский потенциал не является абсолютно прозрачным.

3. Случай абсолютной непроницаемости кулоновского потенциала соответствует выбору

$$
T_{\mathrm{R}}(k)=0 .
$$

В этом случае

$$
\begin{gathered}
R_{\mathrm{R}}(k)=\frac{\Gamma(i \alpha /(2 k))}{\Gamma(-i \alpha /(2 k))}, \\
\left|R_{\mathrm{R}}(k)\right|^{2}=1 .
\end{gathered}
$$

Значения коэффициентов рассеяния (75) и (76) реализуются в трех случаях: при связи

$$
v_{+}^{-, V}=-\frac{2+\left(1-e^{\pi \alpha / k}\right) v_{+}^{+, W}}{1+e^{\pi \alpha / k}},
$$

или при значениях

$$
v_{+}^{+, W}=-1, \quad v_{-}^{-, V} \neq 1
$$

или

$$
v_{+}^{-, V}=-1, \quad v_{-}^{-, V} \neq 1
$$

Величины двух (а фактически, с учетом соотношения (53), одного) параметров $v_{i}$ остаются произвольными, но это оказьвается несушественным: во всех трех случаях 
(78)-(80) независимо от значения оставшихся параметров коэффициенты в сингулярной добавке $\delta V_{f_{2}}(k, x)(48)$ на положительной полуоси обращаются в бесконечность. Таким образом, случай полной непрозрачности кулоновского потенциала может в принципе рассматриваться, хотя это рассмотрение и носит достаточно формальный характер. Этот случай соответствует решению граничной задачи Дирихле (см., например, [4]), т.е. разделению оси на два независимых промежутка. При этом, очевидно, в качестве решения уравнения (47) разумно рассматривать перенормированную волновую функцию, определяемую условием

$$
f_{2}(k, x)=\mathcal{A}_{\mathrm{R}}(k) f_{2}^{(\mathrm{R})}(k, x),
$$

т.e.

$$
f_{2}^{(\mathrm{R})}(k, x)=\left[\psi_{+}^{-, V}(k, x)+\frac{\Gamma(i \alpha /(2 k))}{\Gamma(-i \alpha /(2 k))} \psi_{+}^{+, W}(k, x)\right] \theta(x),
$$

причем функция $f_{2}^{(\mathrm{R})}(k, x)(82)$ удовлетворяет граничному условию Дирихле

$$
f_{2}^{(\mathrm{R})}(k, 0)=0 .
$$

Подводя итоги, можно констатировать, что предложенный в работе метод позволяет построить с учетом аналитической структуры фундаментальных решений трехпараметрическое семейство решений (самосопряженных расширений), полностью определяющих коэффициенты рассеяния, волновые функции и поправки к кулоновскому потенциалу, а также вычислить соответствуюшие уровни энергии. При этом все указанные величины оказываются однозначно связанными, взаимозависимыми. Кроме того, оказывается, что поправки к потенциалу должны присутствовать обязательно при любых допустимых значениях параметров. Обрашает на себя внимание также замечательное сходство членов $(59),(60)$ с контрчленами, полученными методом размерной регуляризации (причем без введения параметра, нарушаюшего в размерной регуляризации масштабную инвариантность (см., например, [23])).

Таким образом, можно говорить, что уже на уровне расчетов, связанных с нерелятивистским уравнением Шредингера, обязательно присутствуют перенормировки и члены, соответствующие радиационным поправкам КЭД, причем эти величины возникают как проявление внутренней структуры соответствующего динамического уравнения. Сушественно, что эти величины можно вычислять, не прибегая к методам теории возмушений.

В целом предложенньй метод указывает на возможность интерпретации широкого класса явлений квантовой теории как результата расширения соответствующего оператора Гамильтона до самосопряженного, а в части, касаюшейся теории перенормировок, может рассматриваться как обобщение метода перенормировок Боголюбова, Парасюка и Хеппа.

Заметим, наконец, что в данной работе получено решение, соответствующее четному продолжению волновых функций через точку сингулярности. Вопросы, связанные с проблемой вырождения (и в частности с возможностью нечетного продолжения волновой функции в нуле), с возможностью включения в гамильтониан $\delta^{\prime}$-членов, детальный анализ спектра связанных состояний (включая проблему отсутствия уровня с бесконечно большой отрицательной энергией) в данной работе не рассматривались. Эти проблемы будут проанализированы в следуюшей публикации. 


\section{ПРИЛОЖЕНИЕ}

Используемый в работе математический аппарат в значительной степени разбросан по различным источникам. Отсутствует также и единьй стандарт в описании уравнений с особыми точками. $\mathrm{K}$ тому же ряд формул, использованных в данной работе, не удалось найти в известных математических пособиях. Поэтому приведем краткую сводку использованных в работе математических соотношений. Обозначения в основном соответствуют принятым в книгах [18] и [24].

Уравнение типа (1), (2) получается из вырожденного гипергеометрического уравнения

$$
z \frac{d^{2} w(z)}{d z^{2}}+(c-z) \frac{d w(z)}{d z}-a w(z)=0, \quad a, c, z \in \mathbb{C},
$$

при исключении члена с первой производной. При этом оно приобретает вид

$$
\frac{d^{2} W(z)}{d z^{2}}=\left(\frac{1}{4}-\frac{p}{z}+\frac{m^{2}-1 / 4}{z^{2}}\right) W(z),
$$

где $p=c / 2-a, m=c / 2-1 / 2, W(z)=e^{-z / 2} z^{m+1 / 2} w(z)$, и называется уравнением Уиттекера.

Уравнение (П.1) имеет регулярную особую точку в начале координат с показателями 0 и $1-c$ и иррегулярную особую точку ранга 1 на бесконечности.

Решение в виде ряда уравнения (П.1), соответствуюшее показателю 0 в точке $z=0$, имеет вид

$$
M(a, c, z)=\sum_{s=0}^{\infty} \frac{(a)_{s}}{(c)_{s}} \frac{z^{s}}{s !}, \quad c \neq 0,-1,-2, \ldots
$$

$\left((a)_{s},(c)_{s}\right.$ - символы Похгаммера). Этот ряд сходится при всех конечных $z$ и определяет функцию Куммера. Функция $M(a, c, z)$ является в общем случае мероморфной функцией переменной $c$ с полюсами в точках $0,-1,-2, \ldots$ и целой по $a$. Второму показателю $1-c$ при $z=0$ соответствует решение

$$
N(a, c, z)=z^{1-c} M(1+a-c, 2-c, z), \quad c \neq 2,3,4, \ldots .
$$

Определяя вронскиан двух функций стандартным образом:

$$
\mathcal{W}\left\{w_{1}(z), w_{2}(z)\right\}=w_{1}(z) w_{2}^{\prime}(z)-w_{1}^{\prime}(z) w_{2}(z),
$$

легко получить, что определитель Вронского функций (П.3) и (П.4) имеет вид

$$
\mathcal{W}\{M(a, c, z), N(a, c, z)\}=(1-c) e^{z} z^{-c} .
$$

Парой линейно независимых при всех значениях параметров решений уравнения (П.1), соответствующих иррегулярной особой точке, являются функции $U(a, c, z)$ и $V(a, c, z)$, имеющие нормальные разложения вида

$$
\begin{aligned}
& U(a, c, z) \sim z^{-a} \sum_{s=0}^{\infty}(-1)^{s} \frac{(a)_{s}(1+a-c)_{s}}{s ! z^{s}}, \quad z \rightarrow \infty, \quad|\arg z| \leqslant \frac{3 \pi}{2}-\delta, \\
& V(a, c, z) \sim e^{z}(-z)^{a-c} \sum_{s=0}^{\infty} \frac{(c-a)_{s}(1-a)_{s}}{s ! z^{s}}, \quad z \rightarrow \infty,|\arg (-z)| \leqslant \frac{\pi}{2}-\delta,
\end{aligned}
$$


где $\delta$ - произвольная малая положительная постоянная. $U(a, c, z)$ и $V(a, c, z)$ - многозначные функции $z$, целые по $a$ и $c$. Функции $U(a, c, z)$ и $V(a, c, z)$ связаны между собой соотношением

$$
V(a, c, z)=e^{z} U(c-a, c,-z) .
$$

Определитель Вронского для функций $U(a, c, z)$ и $V(a, c, z)$ имеет вид

$$
\mathcal{W}\{U(a, c, z), V(a, c, z)\}=e^{\varepsilon(\operatorname{Im} z) i \pi(c-a)} e^{z} z^{-c},
$$

где $\varepsilon(\operatorname{Im} z)$ определяется формулой (3).

Стандартные решения уравнения (П.2) имеют вид

$$
\begin{aligned}
& M_{p, m}(z)=e^{-z / 2} z^{m+1 / 2} M\left(m-p+\frac{1}{2}, 2 m+1, z\right), \\
& W_{p, m}(z)=e^{-z / 2} z^{m+1 / 2} U\left(m-p+\frac{1}{2}, 2 m+1, z\right)
\end{aligned}
$$

и называются функциями Уиттекера. Каждое из решений (П.11) и (П.12) является многозначной функцией $z$. Главные ветви соответствуют области $\arg z \in(-\pi, \pi]$. Асимптотическое поведение решений (П.11) и (П.12) определяется выражениями

$$
\begin{aligned}
& M_{p, m}(z) \sim z^{m+1 / 2}, \quad z \rightarrow 0, \quad 2 m \neq-1,-2,-3, \ldots, \\
& W_{p, m}(z) \sim e^{-z / 2} z^{p}, \quad z \rightarrow \infty, \quad|\arg z| \leqslant \frac{3 \pi}{2}-\delta .
\end{aligned}
$$

Поскольку в работе исследуется задача рассеяния, предпочтительно рассматривать решения, обладающие асимптотическим поведением типа (П.14). Поэтому в качестве фундаментальной пары будем использовать решения, связанные с функциями

$$
\begin{gathered}
W_{p, m}(z)=e^{-z / 2} z^{m+1 / 2} U\left(m-p+\frac{1}{2}, 2 m+1, z\right), \\
V_{p, m}(z)=e^{-z / 2} z^{m+1 / 2} V\left(m-p+\frac{1}{2}, 2 m+1, z\right) .
\end{gathered}
$$

Из разложений (П.7) и (П.8) и формулы (П.9) видно, что при $z \rightarrow \infty$

$$
V_{p, m}(z) \sim e^{z / 2} z^{-p}
$$

Из сравнения уравнения Уиттекера (П.2) и стационарного одномерного уравнения Шредингера (1) с потенциалом (2) видно, что при

$$
p=-\frac{i \alpha}{2 k}, \quad m=\frac{1}{2}, \quad z=-2 i k x
$$

фундаментальная пара решений уравнения (1), (2), соответствуюшая задаче рассеяния, при $x>0$ имеет вид (4a), (4б). Как видно из выражения (2), переход на отрицательную полуось соответствует замене $\alpha \rightarrow-\alpha$ при $x<0$, что приводит к паре решений (5a), (5б). Нормировочные множители, выписанные в выражениях (4a)-(5б), приводят к асимптотическим разложениям (6a)-(6г). 
Подстановка (П.18), приводящая уравнение Уиттекера (П.2) к виду (1), (2), соответствует тому, что параметр $c$ в функциях $U(a, c, z), V(a, c, z)$ имеет целочисленное значение $(c=2)$. Это приводит к необходимости использовать предельные формы функций $M(a, c, z)$ и $N(a, c, z)$ (получаемые, например, с помощью метода Фробениуса) для описания поведения $U(a, c, z)$ и $V(a, c, z)$ около точки $z=0$. Соответствующее логарифмическое разложение для функции $U(a, c, z)$ при $c=r(r \in \mathbb{Z})$ имеет в общем случае вид

$$
U(a, r, z)=\sum_{s=1}^{r-1}(-1)^{s-1} \lambda_{r,-s} \frac{(s-1) !}{z^{s}}+\lambda_{r, 0} M(a, r, z) \ln z+\sum_{s=0}^{\infty} \lambda_{r, s} \mu_{r, s} \frac{z^{s}}{s !}
$$

где

$$
\begin{aligned}
\lambda_{r, s} & =\frac{(-1)^{r} \Gamma(a+s)}{\Gamma(a) \Gamma(1+a-r)(r+s-1) !}, \\
\mu_{r, s} & =\psi(a+s)-\psi(1+s)-\psi(r+s),
\end{aligned}
$$

$\psi(z)=\Gamma^{\prime}(z) / \Gamma(z)$ - дигамма-функция. Подстановка значений (П.18) дает для фундаментальной пары решений (4a), (4б) и их производных с точностью до членов второго порядка по $z$ разложения

$$
\begin{aligned}
& \psi_{+}^{+, W}(k, x)=\frac{2 k}{\alpha \Gamma(i \alpha /(2 k))} e^{\pi \alpha /(4 k)}\left\{-i+x\left[i \alpha-2 i \alpha \gamma_{\mathrm{E}}+k-i \alpha \ln (-2 i k)-\right.\right. \\
& \left.\left.\quad-i \alpha \psi\left(1+\frac{i \alpha}{2 k}\right)-i \alpha \ln x\right]\right\}+O\left(x^{2}\right), \\
& {\left[\psi_{+}^{+, W}(k, x)\right]^{\prime}=\frac{2 k}{\alpha \Gamma(i \alpha /(2 k))} e^{\pi \alpha /(4 k)}\left\{-2 i \alpha \gamma_{\mathrm{E}}+k-i \alpha \ln (-2 i k)-i \alpha \psi\left(1+\frac{i \alpha}{2 k}\right)-\right.} \\
& \quad-i \alpha \ln x+x\left[2 i \alpha^{2}-2 i \alpha^{2} \gamma_{\mathrm{E}}+3 \alpha k+i k^{2}-i \alpha^{2} \ln (-2 i k)+2 \alpha k \psi\left(1+\frac{i \alpha}{2 k}\right)-\right. \\
& \left.\left.\quad-i \alpha^{2} \psi\left(2+\frac{i \alpha}{2 k}\right)-2 \alpha k \psi\left(2+\frac{i \alpha}{2 k}\right)-i \alpha^{2} \ln x\right]\right\}+O\left(x^{2}\right), \\
& \psi_{+}^{-, V}(k, x)=-\frac{2 k}{\alpha \Gamma(-i \alpha /(2 k))} e^{\pi \alpha /(4 k)}\left\{-i+x\left[i \alpha-2 i \alpha \gamma_{\mathrm{E}}-k-i \alpha \ln (2 i k)-\right.\right. \\
& \left.\left.\quad-i \alpha \psi\left(1-\frac{i \alpha}{2 k}\right)-i \alpha \ln x\right]\right\}+O\left(x^{2}\right), \\
& {\left[\psi_{+}^{-, V}(k, x)\right]^{\prime}=-\frac{23)}{\alpha \Gamma(-i \alpha /(2 k))} e^{\pi \alpha /(4 k)}\left\{-2 i \alpha \gamma_{\mathrm{E}}-k-i \alpha \ln (2 i k)-i \alpha \psi\left(1-\frac{i \alpha}{2 k}\right)-\right.} \\
& \quad-i \alpha \ln x+x\left[2 i \alpha^{2}-2 i \alpha^{2} \gamma_{\mathrm{E}}-3 \alpha k+i k^{2}-i \alpha^{2} \ln (2 i k)-2 \alpha k \psi\left(1-\frac{i \alpha}{2 k}\right)-\right. \\
& \left.\left.\quad-i \alpha^{2} \psi\left(2-\frac{i \alpha}{2 k}\right)+2 \alpha k \psi\left(2-\frac{i \alpha}{2 k}\right)-i \alpha^{2} \ln x\right]\right\}+O\left(x^{2}\right) .
\end{aligned}
$$

Как было указано выше, замена $\alpha \rightarrow-\alpha$ дает соответствуюшие разложения для фундаментальной пары $(5 \mathrm{a}),(5 б)$. 
Ранее уже отмечалось, что $U(a, c, z)$ и $V(a, c, z)$ - многозначные функции $z$. Используя соотношения

$$
\begin{aligned}
W_{p, m}(z) & =\frac{\Gamma(-2 m)}{\Gamma(1 / 2-m-p)} M_{p, m}(z)+\frac{\Gamma(2 m)}{\Gamma(1 / 2+m-p)} M_{p,-m}(z), \\
V_{p, m}(z) & =\frac{\Gamma(-2 m)}{\Gamma(1 / 2-m+p)} M_{p, m}(z)+\frac{\Gamma(2 m) e^{-2 i \pi m}}{\Gamma(1 / 2+m+p)} M_{p,-m}(z),
\end{aligned}
$$

а также учитьвая, что

$$
M_{-p, m}\left(z e^{i \pi}\right)=i e^{i \pi m} M_{p, m}(z),
$$

можно получить формулы для аналитических продолжений (при $s \in \mathbb{Z}$ )

$$
\begin{aligned}
(-1)^{s} W_{p, m}\left(z e^{2 i \pi s}\right)= & -\frac{e^{2 i \pi p} \sin (2 \pi s m)+\sin [2 \pi(s-1) m]}{\sin (2 \pi m)} W_{p, m}(z)+ \\
& +\frac{\sin (2 \pi s m)}{\sin (2 \pi m)} \frac{2 \pi e^{i \pi(p+m)}}{\Gamma(1 / 2+m-p) \Gamma(1 / 2-m-p)} V_{p, m}(z), \\
(-1)^{s} V_{p, m}\left(z e^{2 i \pi s}\right)= & -\frac{e^{2 i \pi p} \sin (2 \pi s m)+\sin [2 \pi(s+1) m]}{\sin (2 \pi m)} V_{p, m}(z)- \\
& -\frac{\sin (2 \pi s m)}{\sin (2 \pi m)} \frac{2 \pi e^{i \pi(p-m)}}{\Gamma(1 / 2-m+p) \Gamma(1 / 2+m+p)} W_{p, m}(z) .
\end{aligned}
$$

В рассматриваемом случае следует, естественно, использовать предельные формы выражений для $U(a, c, z)$ и $V(a, c, z)$.

Кроме приведенных в основном тексте вронскианов $(7),(8)$, в работе также используются соотношения

$$
\begin{aligned}
\mathcal{W}\left\{\left[\psi_{+}^{-, V}(k, x)\right]^{*}, \psi_{+}^{-, V}(k, x)\right\} & =-2 i k, \\
\mathcal{W}\left\{\left[\psi_{+}^{+, W}(k, x)\right]^{*}, \psi_{+}^{+, W}(k, x)\right\} & =2 i k, \\
\mathcal{W}\left\{\left[\psi_{+}^{-}, V(k, x)\right]^{*}, \psi_{+}^{+, W}(k, x)\right\} & =0,
\end{aligned}
$$

причем

$$
\left[\psi_{+}^{-, V}(k, x)\right]^{*}=\psi_{+}^{+, W}(k, x) .
$$

При анализе выражения (59) в работе используется разложение в ряд функции $\operatorname{Re} \psi(z)$. При $z=x+i y$

$$
\begin{aligned}
\operatorname{Re} \psi(i y) & =\operatorname{Re} \psi(-i y)=\operatorname{Re} \psi(1+i y)=\operatorname{Re} \psi(1-i y)= \\
& =-\gamma_{\mathrm{E}}+y^{2} \sum_{n=1}^{\infty} \frac{1}{n} \frac{1}{n^{2}+y^{2}}, \quad-\infty<y<\infty
\end{aligned}
$$

Асимптотические формулы для $\operatorname{Re} \psi(i y)$ имеют вид

$$
\operatorname{Re} \psi(i y) \sim \ln y+\sum_{n=1}^{\infty} \frac{(-1)^{n-1} B_{2 n}}{2 n y^{2 n}}=\ln y+\frac{1}{12 y^{2}}+\frac{1}{120 y^{4}}+\frac{1}{252 y^{6}}+\cdots, \quad y \rightarrow \infty
$$

$\left(B_{2 n}\right.$ - числа Бернулли). 
Благодарности. Автор считает своим приятным долгом выразить искреннюю признательность Б. А. Арбузову, В.И. Саврину и В.Е. Троищкому за исключительно ценную помощь и полезные обсуждения.

\section{Список литературы}

[1] K. M. Case. Phys. Rev. 1950. V. 80. P. 797.

[2] R. de L. Kronig, W. G. Penney. Proc. Roy. Soc. A. 1931. V. 130. P. 499.

[3] W. M. Frank, D. J. Land, R. M. Spector. Rev. Mod. Phys. 1971. V. 43. P. 36; Ю. Н. Демков, B. Н. Островский. Метод потенциалов нулевого радиуса в атомной физике. Л.: ЛГУ, 1975.

[4] С. Альбеверио, Ф. Гестези, Р. Хеәг-Крон, Х. Холден. Решаемые модели в квантовой механике. М.: Мир, 1991.

[5] J. Zorbas. J. Math. Phys. 1980. V. 21. P. 840; S. Albeverio, F. Gesztesy, R. Høegh-Krohn, L. Streit. Ann. Inst. H. Poincare A. 1983. V. 38. P. 263.

[6] F. Rellich. Math. Z. 1943/44. V. 49. P. 702.

[7] Ф. А. Березин, Л. Д. Фаддеев. ДАН СССР. 1961. Т. 137. С. 1011.

[8] M. Moshinsky. J. Phys. A. 1993. V. 26. P. 2245.

[9] R. G. Newton. J. Phys. A. 1994. V. 27. P. 4717.

[10] M. Moshinsky. J. Phys. A. 1994. V. 27. P. 4719.

[11] М. Рид, Б. Саймон. Методы современной математической физики. Т. 1. М.: Мир, 1977.

[12] М. Рид, Б. Саймон. Методы современной математической физики. Т. 2. М.: Мир, 1978.

[13] E. H. Lieb, W. Liniger. Phys. Rev. 1963. V. 130. P. 1605; E. H. Lieb. Phys. Rev. 1963. V. 130. P. 1616

[14] C. Aneziris, A.P. Balachandran, D. Sen. Int. J. Mod. Phys. A. 1991. V. 6. P. 4721; Erratum. 1992. V. 7. P. 1851; A. P. Balachandran. Int. J. Mod. Phys. B. 1991. V. 5. P. 2585.

[15] M. Carreau, E. Farhi, S. Gutmann. Phys. Rev. D. 1990. V. 42. P. 1194; M. Carreau. J. Phys. A. 1993. V. 26. P. 427.

[16] F. A. B. Coutinho, Y. Nogami, J. F. Perez. J. Phys. A. 1999. V. 32. P. L133.

[17] Ф. А. Березин, М. А. Шубин. Уравнение Шредингера. М.: Изд-во МГУ, 1983.

[18] $\Phi$. Олвер. Асимптотики и специальные функции. М.: Наука, 1990.

[19] А.И. Базь, Я. Б. Зельдович, А. М. Переломов. Рассеяние, реакции и распады в нерелятивистской квантовой механике. М.: Наука, 1971.

[20] N. N. Bogoliubov, O.S. Parasiuk. Acta Math. 1957. V. 97. P. 227; K. Hepp. Commun. Math. Phys. 1966. V. 2. P. 301.

[21] Н. Н. Боголюбов, Д. В. Ширков. Введение в теорию квантованных полей. М.: Наука, 1973.

[22] Дж. Д. Бьеркен, С. Д. Дрелл. Релятивистская квантовая теория. Т. 1. М.: Наука, 1978.

[23] Ф. Индурайн. Квантовая хромодинамика. М.: Мир, 1986; R. M. Barnett, M. Dine, L. McLerran. Phys. Rev. D. 1980. V. 22. P. 594.

[24] М. Абрамович, И. М. Стиган (ред.). Справочник по специальным функциям. М.: Наука, 1979.

Поступила в редакцию $18 . I V .2003$ г., после доработки 29.VIII.2003 г. 